\title{
Catalog of averaged magnetic phase curves of stars
}

\section{Second edition ${ }^{\star}$}

\author{
V. D. Bychkov ${ }^{1}$, L. V. Bychkova ${ }^{1}$, and J. Madej ${ }^{2}$ (] \\ 1 Special Astrophysical Observatory of RAS, Nizhnij Arkhyz 369167, Russia \\ e-mail: vbych@sao.ru \\ 2 Astronomical Observatory, University of Warsaw, Al. Ujazdowskie 4, 00-478 Warszawa, Poland \\ e-mail: jm@astrouw.edu.pl
}

Received 22 December 2020 / Accepted 21 April 2021

\begin{abstract}
Some stars show variability of the longitudinal components of their global magnetic fields, mainly resulting from rotation. Recently, a lot of new observational data have accumulated for various types of stars. To summarize information about magnetic variability, we present the second version of the catalog of average magnetic phase curves. The curves are obtained from literary data and as a result of observations from SAO RAS telescopes. A total of 357 stars of various spectral types were studied based on data that was published before the end of August 2020. Magnetic Ap and Bp stars make up the most numerous subset in the catalog (224 objects). The phase curves were obtained by least-squares fit of the simple sine wave or double sine wave, which are presented in uniform form. The parameters of variability of these objects are presented in tabular form; the upper limits of observed magnetic fields of various types are indicated. The information is presented in a convenient form for verification of various kinds of theoretical models. For clarity and ease of use, phase dependences are presented in graphic form in 514 figures. Periods of variability are defined or refined for a number of stars. The created catalog allows the estimation of the limits of the observed magnetic field variability for various types of stars and the estimation of the energy of the magnetic fields of stars. The catalog will be useful for the development of observation programs. The catalog facilitates the study of the orientation of the axes of rotation of stars in space, the investigation of the interactions of magnetic fields of stars with the interstellar medium, and the "magnetic braking" mechanism. This catalog also permits the study of the orientation of the orbits of planets for some stars with planets and planetary systems.
\end{abstract}

Key words. stars: magnetic field - stars: rotation - stars: fundamental parameters

\section{Introduction}

The global magnetic fields (MFs) of stars are typically studied by measuring the Zeeman splitting of spectral lines in circularly polarized light (Babcock 1947). The resulting observable quantity is the longitudinal (effective) $\mathrm{MF}, B_{\mathrm{e}}$, which usually exhibits periodic variations caused by the rotation of a star, which was noted in the first Babcock Magnetic Survey Catalog (Babcock 1958). Apparent magnetic variations of this type were analysed by Stibbs (1950), who studied the effect of the rotation of the dipole MF on the displacement of the Zeeman components in a spectral line.

Longitudinal MF strength, $B_{\mathrm{e}}$, is a projection of the local vector of the surface stellar MF on the line of sight integrated over the visible stellar disk; see Babcock (1947) and the appendix in Stibbs (1950). Periodic variations of $B_{\mathrm{e}}$ are caused by the rotation of the star because, commonly the field configuration is not symmetrical about the axis of rotation (as in the inclined dipole model). The period of the apparent magnetic $B_{\mathrm{e}}$ variations is then equal to the rotational period.

Periodic variability of the longitudinal MF correlated with the rotation is a purely geometrical effect and it commonly occurs when the true surface MF is intrinsically constant during

\footnotetext{
* All appendices are only available at the CDS via anonymous ftp to cdsarc.u-strasbg. fr $(130.79 .128 .5)$ or via http://cdsarc. u-strasbg.fr/viz-bin/cat/J/A+A/652/A31
}

the rotational period (Babcock 1960). Intrinsic variations of the surface MF (owing to solar-like cycles) are not considered in this work. For example, we neglect physical changes to the global MFs of M dwarfs, which proceed over a timescale of one year, while presenting $B_{\text {e }}$ periodic variations, which proceed within the timescale of the rotation (several days).

Another observable scalar magnetic quantity is the surface $\mathrm{MF}, B_{\mathrm{s}}$, which can be measured from the separation of Zeemansplit line components seen in natural light in high-dispersion spectra of stars with magnetically resolved line components; see Babcock (1960) and Preston (1971).

Both observables, $B_{\mathrm{e}}$ and $B_{\mathrm{s}}$, were defined and applied to observations of spectral lines in early-type stars over 60 years ago, when the available instruments and observational techniques were much simpler or less advanced than now. Since then, the growing interest in stellar magnetism caused the inflow of new magnetic observational data for stars of various spectral types, which also was possible using new high-resolution spectropolarimeters, which were very productive in this area.

\section{New magnetic measurements}

The situation with magnetic measurements for the last 15 years has changed significantly primarily owing to the increased accuracy and number of measurements. Therefore, in the period from 2005 to 2019, more than $30000 \mathrm{MF}$ estimates were received. 
Table 1. Number of stars in various classes with known MPCs.

\begin{tabular}{lrlllr}
\hline \hline Ap/Bp & 224 & Stars hosting planets & 8 & T Tau stars & 6 \\
Var. $\beta$ Cep type & 16 & Normal chem. comp.stars & 5 & Var. Ori type & 3 \\
Slowly pulsating B stars & 9 & Be stars & 6 & Pre-main sequence & 3 \\
High proper motion stars & 14 & Var. $\delta$ Sct type & 3 & Rotationally var. stars & 18 \\
Var. $\delta$ Cep type & 1 & Semi-regular var.pulsating & 2 & EB Algol type & 1 \\
Multiple stars & 19 & Flare stars & 17 & Var. RS CVn type & 2 \\
Pulsating stars & 5 & Ae/Be Herbig stars & 10 & Blue supergiant star & 2 \\
Var. BY Dra & 8 & & & \\
\hline
\end{tabular}

The greatest number of new high-precision measurements were obtained in the last few years using the following telescopes: (1) Approximately $38.5 \%$ were obtained using the Echelle SpectroPolarimetric Device for the Observation of Stars (ESPaDonS) at the $3.6 \mathrm{~m}$ Canada-France-Hawaii Telescope (CFHT). (2) Approximately $23.1 \%$ were obtained using the FOcal Reducer and low dispersion Spectrograph 1 or 2 (FORS1/2) at the ESO $8 \mathrm{~m}$ telescopes UT1, UT2 or UT3 (ESO). (3) About $17.7 \%$ of data were obtained using NARVAL, a copy of the ESPaDOnS, at the $2 \mathrm{~m}$ Telescope Bernard Lyot (TBL), Observatoire du Pic du Midi in the French Pyrenees. (4) Moreover, 7.4\% were obtained with the High Accuracy Radial velocity Planet Searcher (HARPS) at the $3.6 \mathrm{~m}$ telescope, La Silla (ESO), (5) Roughly 6.3\% with Main Stellar Spectrograph (MSS), which is permanently installed at the $6 \mathrm{~m}$ telescope at the Special Astrophysical Observatory of the Russian Academy of Sciences (SAO RAS), and 3.1\% with the MultiSite Continuous Spectroscopy) spectropolarimeter (MuSiCoS) at the $2 \mathrm{~m}$ TBL at the Pic du Midi, France, at the $2.5 \mathrm{~m}$ Isaac Newton Telescope (La Palma) and the $1.9 \mathrm{~m}$ telescope of the South African Astronomical Observatory (SAAO). The remaining measurements were obtained using a number of other instruments, but the contribution of each did not exceed $1 \%$. Naturally, this only displays the current state.

Thanks to these measurements it was possible to specify already known MPC and also make new observations. Therefore, out of 136 stars of the first catalog, only 23 MPCs remained unchanged. The remaining 113 were supplemented by new measurements, and therefore new parameters were obtained for their MF variability. The MF estimates obtained with low accuracy (mostly photographic) measurements were often discarded and only highly accurate estimates obtained by modern methods were used. For example, for building an MPC in the first edition of the catalog relatively low-resolution photographic MF estimates amounted to $54 \%$ and to only $6.6 \%$ for the MPC of the second catalog.

Table 1 lists the principal types of stars included in the catalog of magnetic phase curves (MPCs). We designate B stars with development circumstellar disks in Table 1 under Be stars. Some stars were simultaneously put into two different classes. For example, HD 96446 belongs to He-r and $\beta$ Cep classes, HD 97048 belongs to TTS and Ae/Be Herbig types, HD 5737 is a B6pSrTi He-w and rotationally variable star. Binary system DT Vir consists of two companions: UV and RS (flare and RS CVn type stars). These stars are counted simultaneously in each type in the appendices.

Table 1 provides data on the number of stars each type. The second catalog expanded not only in the number of stars, but also in the types of stars. It contains MPC information for flare stars, multiple stars, hosting planets stars, Be stars, and a number of other types of stars that were not in the first catalog. For some types of stars, the number of MPCs was noticeable and increased, allowing us to arrive at more confident conclusions about the variability of MFs in these stars.

The number of MPCs for CP stars increased from 127 to 224 in the new catalog. However, most importantly, the higher accuracy allowed us to identify more deviations from purely harmonic phase dependences. The percentage of stars with double sine wave curves increased from the first catalog with 18 out of $127(14 \%)$ to the second catalog with 62 out of 224 (25\%). This is very important for the study of the origin and evolution of MF stars.

In the first catalog we noted a noticeable deviation of the $B_{0}$ coefficient from zero for 18 Ap stars with a double sine wave (the average $B_{0}=-473 \pm 296 \mathrm{G}$ ). This observation was confirmed in the second catalog containing 49 Ap stars, where the average $\left(B_{0}=-250 \pm 206 \mathrm{G}\right)$. We note that in the second directory the unchanged MPCs (and their parameters) remain only for 23 stars (7\%) from the first catalog.

The high number of new, precise MF measurements and the recent discovery of the global MF in stars of various types motivated us to compile and submit the entire second catalog rather than just an addition to the first. The larger second edition also represents an expansion into previously unexplored classes of stellar magnetic activity.

\section{The second catalog}

The first edition of this catalog (Bychkov et al. 2005) presented MPCs, $B_{\mathrm{e}}(\phi)$, for 136 stars, most of which were Ap/Bp or related stars in the main sequence with strong surface fields. Recently, the application of the newest instrumentation and data reduction procedures has allowed the detection of global MFs and the partial investigation of their behaviors among stars of many other spectral classes.

In this work, we present and analyse the results of longitudinal MF measurements, $B_{\mathrm{e}}$, and data on the surface field, $B_{\mathrm{s}}$, for 357 stars. The data were obtained from various bibliographic sources and originally were measured using both old photographic plates and the newest high-resolution spectrographs. We appended data compiled from personal communications, as well as our own determinations obtained at the Special Astrophysical Observatory (Russian Academy of Sciences) and magnetic measurements of stars in the Orion OB1 Association (Borra 1981) by permission of Dr. E. F. Borra.

For a number of stars, we represent not one, but several MPCs. This is because MPCs can be derived from measurements on various elements. So for Ap stars, the MPCs obtained through lines of hydrogen and metals can differ noticeably. The MPCs obtained on various chemical elements can also differ greatly from one another. This difference arises from the heterogeneous distribution of chemical elements along the surface of the star 
and the position of areas (spots) with increased concentration of the corresponding chemical element relative to the magnetic pole or the equator. For other types of stars, for example, for later active flare stars, MPCs can vary significantly from set to the set because the global MF of these stars can change significantly for several months in time. The parameters of each MPC are presented in Tables A.2-A.5 (available at the CDS).

In this catalog Ap and Bp stars are still the most wellinvestigated group of stars; in this work, the MPCs for 224 objects of this type are collected. We did not include white dwarf stars or other degenerate objects and selected only stars built of normal matter.

The catalog of MPCs in the present version can be applied to review and compare the magnetic behaviours of a wide range of types. The MPCs and their parameters are presented in homogeneous graphical and numerical forms, respectively.

\section{Averaged magnetic rotational phase curves}

Periodic variations of the longitudinal (effective) or surface MF, $B_{\mathrm{e}}$ or $B_{\mathrm{s}}$, with the rotational phase, $\phi$, were approximated by the two lowest terms in the Fourier series expansion.

1. For all stars with an adequate number of $B_{\mathrm{e}}$ determinations and for which the period of magnetic variability, $P_{\mathrm{mag}}$, is known, we determined the best fit for $B_{\mathrm{e}}$ versus phase as follows:

$B_{e i}(\phi)=B_{0}+B_{1} \cos (\phi-\pi)$,

where

$\phi=2 \pi\left(\frac{T_{i}-T_{0}}{P}\right)$,

using the least-squares method. Here, $B_{0}$ is the average field and $B_{1}$ equals half the amplitude, $T_{i}$ is the time at which the measurement was taken, $P$ denotes the period, and $T_{0}$ is the time corresponding to the zero phase, $\phi$. The half-amplitudes $B_{1}$ in Eq. (1) are positive numbers by the assumption. We selected the zero epoch, $T_{0}$, in such a way that the phase $\phi=0$ corresponds to the minimum of the best fit MPC.

2. In cases in which the shape of the MPCs is more complex than a simple cosine, we included the second harmonic wave,

$B_{e i}(\phi)=B_{0}+B_{1} \cos \left(\phi+z_{1}\right)+B_{2} \cos \left(2 \phi+z_{2}\right)$,

where phases $z_{1}$ and $z_{2}$ are also parameters of fitting. The halfamplitudes $B_{2}$ can be either positive or negative.

3. The most important variable parameter is the period. We usually sought to use periods taken from the literature. Mostly these are photometric periods, but sometimes we determined or clarified the periods themselves using standard methods, as in the work of Bychkov et al. (2016). In some cases, there are contradictions between photometric and magnetic periods. A typical example is the Ap star HD 5601.

The photometric period determined by Stromgren photometry (Hensberge et al. 1981) $P_{\text {rot }}=1.110 \pm 0.002 \mathrm{~d}$. The photometric period determined by HIPPARCos photometry (Romanyuk et al. 2016) $P_{\text {rot }}=1.756$ d. The photometric period determined by TESS photometry (Sikora et al. 2019) $P_{\text {rot }}=$ $9.85 \pm 0.02 \mathrm{~d}$. From magnetic measurements, we found the period of $P=1.114 \pm 0.003 \mathrm{~d}$ close to the period from photometry (Hensberge et al. 1981). The photometric period is not consistent with magnetic measurements in a number of stars, for example, HD 36540, HD 90569, HD 96707, HD 116114, HD 149822, and others. Naturally, these contradictions will be eliminated as photometric, magnetic, and polarimetric data accumulate. In the commentaries (Appendix B) for each MPC received, the source of the period is indicated; the period is taken from a literary source, which is specified or found by us on magnetic measurements.

We are aware that the periods determined in those cases require additional $B_{\mathrm{e}}$ measurements, or new spectral, photometric, or polarimetric observations to confirm our magnetic periods and reduce their errors. The Tables A.2 and A.5 indicate the sources of periods. Many works are devoted to the problems of searching for periods of rotation of Ap stars (e.g., Mikulášek \& Skarka 2020 and others). There are also observed small variations in the rotation periods of some Ap stars (Mikulášek 2016). This effect should also be taken into account when evaluating the periods of Ap stars. The periods of Ap stars, in which small variations of the period are observed, are indicated in the tables.

4. There are also exceptional stars that exhibit still more complex MPCs, and in such cases Eq. (6) does not provide an adequate fit. A very good example is the star HD 37776, which exhibits the MPC of exceptional complexity. For this star we present various forms of fitting curves and regard this as a unique case.

5. The average MPCs in the catalog were obtained by the least-squares fitting of a sine wave or a double sine wave to the observed $B_{\mathrm{e}}$ points for each star. This is the same method used in the first version of the catalog (Bychkov et al. 2005). In general, this catalog was built following criteria very similar to those adopted for its first version.

Tables A.2-A.5 present the parameters of the MPCs as well as parameter $r$, which was defined by Stibbs (1950). Parameter $r$ relates both the angle $\beta$ between the magnetic dipole axis and the rotational axis, and the angle $i$ between the rotational axis and the line of sight as follows:

$r=\frac{\cos \beta \cos i-\sin \beta \sin i}{\cos \beta \cos i+\sin \beta \sin i}=\frac{B_{\mathrm{e}}(\min )}{B_{\mathrm{e}}(\max )}$.

\section{Discussion: Stars in our catalog}

The following subsections detail the statistical properties of various parameters in the catalog; these include spectral types, rotational periods, and fitting coefficients $B_{0}, B_{1}$, and $B_{2}$.

\subsection{Distribution of spectral and variability types}

Figure 1 shows the distribution of stars in our catalog by spectral types. Magnetic rotational phase curves, $B_{\mathrm{e}}(\phi)$, are determined mostly for stars of early spectral types. Figure 1 shows that the highest concentration of stars is located around the spectral type A.

Therefore, the present knowledge of the global MFs across the Hertzsprung-Russell diagram is still poor and selective, and the completeness of this catalog is weak.

\subsection{Ap/Bp stars}

Ap stars are the most well investigated and numerous subclass in this catalog with 224 stars. This subclass spans a very wide range from late $\mathrm{O}$ to early $\mathrm{F}$ spectral types.

There are 166 Ap stars with simple sine wave phase curves (75\%) and 62 stars with double sine wave MPC (28\%). The proportion of stars with complex MPCs increased with respect to the first edition (Bychkov et al. 2005), which reflects the increased accuracy of the new measurements. 




Fig. 1. Distribution of stars with known $B_{\mathrm{e}}(\phi)$ phase curves by spectral types.

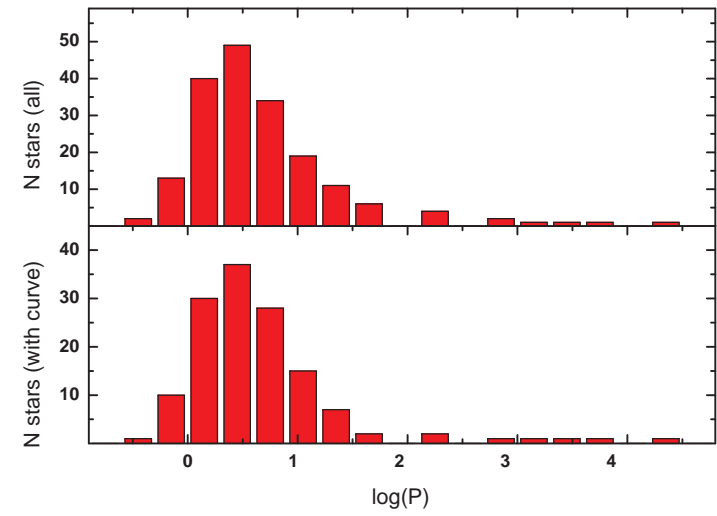

Fig. 2. Distribution of Ap/Bp stars vs. decimal logarithm of period (in days), $\log P_{\text {mag }}$, for all stars with known periods (upper panel) and for stars with known phase curves (lower panel).

\subsubsection{Distribution of periods}

Figure 2 presents the distribution of stars in our catalog versus the decimal logarithm of the period, $P_{\text {mag }}$, separately for all $\mathrm{CP}$ stars with known periods (upper panel) and for stars whose period enables the creation of a rotational phase curves (lower panel). The width of a single bin equals 0.3 dex. The majority of Ap/Bp stars exhibit periods, $P_{\text {mag }}$, between $1 \mathrm{~d}$ and $10 \mathrm{~d}$. The same is true for stars with known phase curves.

\subsubsection{Coefficients $B_{0}, B_{1}$, and $B_{2}$}

Figure 3 shows the number distribution versus coefficient $B_{0}$ for the catalogued stars whose rotational phase curves, $B_{\mathrm{e}}(\phi)$, were approximated by sine waves. Figure 3 does not include HD 215441, whose exceptionally strong $B_{\mathrm{e}}$ field exceeds the scale of the figure. The average value of $B_{0}$ is $+125 \pm 116 \mathrm{G}$. For stars with double sine wave MPCs the average value of $B_{0}$ is $-250 \pm 206 \mathrm{G}$. We conclude that the average longitudinal MF of the MPCs in the catalog, $B_{0}$, equals zero and does not show meaningful bias toward positive or negative values.

Figure 4 represents an analogous number distribution versus coefficient $B_{1}$ (half-amplitude of the $B_{\mathrm{e}}$ variation) for stars with the sine wave rotational phase curves, $B_{\mathrm{e}}(\phi)$. The halfamplitudes, $B_{1}$, usually do not exceed $3000 \mathrm{G}$. The number of

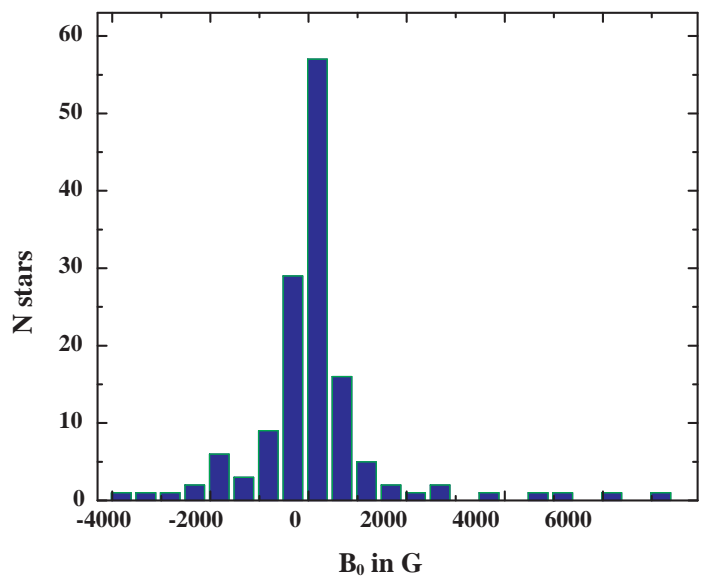

Fig. 3. Distribution of stars vs. the coefficient $B_{0}$ for the subset of stars in which the phase curve $B_{\mathrm{e}}(\phi)$ was approximated by a sine wave.

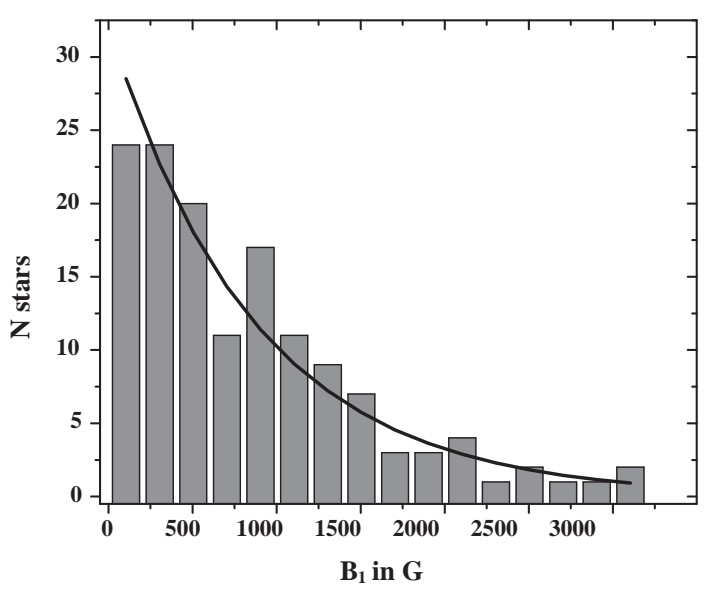

Fig. 4. Number distribution of stars vs. the coefficient $B_{1}$ (halfamplitude of $B_{\mathrm{e}}$ variability). Only stars with sine wave phase curves were included.

stars in a single bin can be approximated by the exponential function,

$n \approx 126.449 \times \exp \left(0.001448 B_{1}\right)$.

We note that the number of stars with the lowest amplitude of $B_{\mathrm{e}}$ variations is underestimated. The accuracy of magnetic measurements is still limited, and many stars with low magnetic variability were not detected as variable stars and therefore were not included in our catalog.

Figure 5 shows an analogous number distribution versus coefficient $B_{1}$ (half amplitude of the $B_{\mathrm{e}}$ variation) for the catalogued stars with double sine wave phase curves, $B_{\mathrm{e}}(\phi)$. The half-amplitudes $B_{1}$ do not usually exceed $5000 \mathrm{G}$. The occurrence of stars in a bin can be approximated by the exponential function,

$n \approx 54.652 \times \exp \left(0.000742 B_{1}\right)$.

For stars with the double sine wave phase curve halfamplitudes $B_{2}$ are usually lower than $B_{1}$ and do not exceed $1200 \mathrm{G}$. The expected number of stars in a bin can be approximated by the exponential function,

$n \approx 109.957 \times \exp \left(0.003044 \operatorname{abs}\left(B_{2}\right)\right)$. 


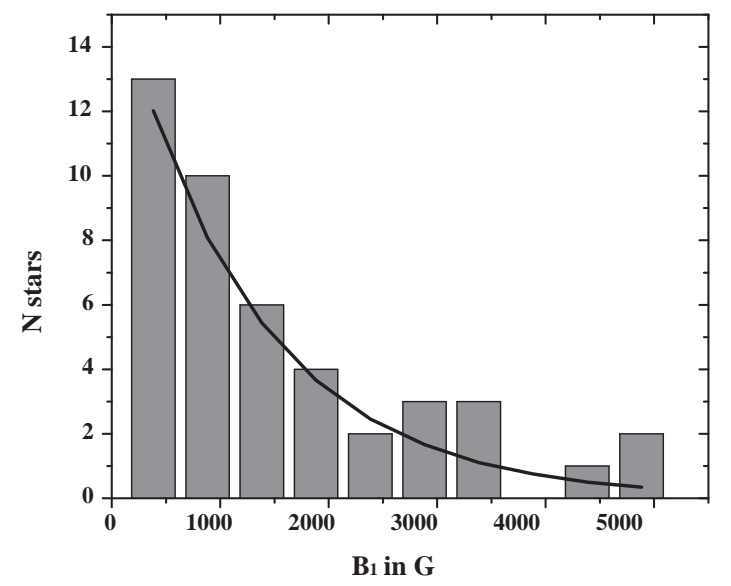

Fig. 5. Number distribution of stars vs. coefficient $B_{1}$ for stars that exhibit a magnetic rotational phase curve with a double sine wave.

\section{Measurements for stars in Orion OB1 association}

In 1994 Dr. E. F. Borra kindly provided us with original estimates of the longitudinal MFs of 13 stars from the Orion OB1 association, which were analysed in his 1981 publication. Measurements were obtained using the Laval University Pockels cell polarimeter, which is similar to the instrument described by Borra \& Landstreet (1980). The author used the Las Campanas $2.5 \mathrm{~m}$ du Pont telescope. The observational techniques and reduction procedures are the same as those reported by Borra \& Landstreet (1980). The results of these measurements are presented in Table A. 6 by permission of Dr. E. F. Borra. We used these estimates in the first catalog (Bychkov et al. 2005) and this second catalog (Borra 1994, priv. comm.).

\section{Contents of appendices}

The appendices present tables, figures, and remaining material in the following manner: Table A.1 lists all stars in the catalog. Tables A.2 and A.3 collected parameters of the phase curves and the magnetic dipole for stars with single wave $B_{\text {e }}$ phase curves and Tables A.4 and A.5 for stars with double wave $B_{\mathrm{e}}$ phase curves. Table A.6 lists $B_{\mathrm{e}}$ measurements for 13 stars in Orion OB1 by EF Borra (priv. comm.). Appendix B presents comments on individual stars, Appendix $\mathrm{C}$ is the cross-reference list, and Appendix D is the collection of the magnetic rotational phase curves (Figs. 1-514).

In some cases several phase curves presented in this work correspond to the same star. This occurred when different observational techniques produced significantly different series of $B_{\mathrm{e}}$ values for that star. Moreover, in a few cases the rotational phase curves of the surface field, $B_{\mathrm{s}}$, are given for stars in which longitudinal MF data were not available.

We note that some entries in Tables A.3 and A.5 present either huge or unrealistically low values of the reduced chisquare for fits to the actual time series. Such a chi-square can imply that the quality of the corresponding MPC is low owing to scattered data points or overestimated errors. On the other hand, we cannot exclude that in some stars the global MF exhibited intrinsic changes during the observing time period. In such cases parameters given in the tables are not of any use.

\section{Summary}

We have compiled a catalog of magnetic rotational phase curves, $B_{\mathrm{e}}(\phi)$, for 357 stars that exhibit periodic variations of the effective MF, $B_{\mathrm{e}}$. Most of the catalogued objects (224 stars) are chemically peculiar A and B type stars. The catalog consists of figures that represent individual $B_{\mathrm{e}}$ measurements, error bars, and phase curves, $B_{\mathrm{e}}(\phi)$, which are approximated either by a sine wave or a double sine wave.

The catalog also presents a listing of the following parameters of MPCs: coefficients $B_{0}, B_{1}$, and $B_{2}$ of the harmonic expansion of $B_{\mathrm{e}}(\phi)$; the magnetic period, $P_{\text {mag }}$ (in days), which is usually equal to the rotational period, $P_{\text {rot }}$; the Julian Day of the zero phase, $T_{0}$; and the coefficient $r$, defined by Stibbs (1950).

There are a number of magnetic stars with strong effective MF, $B_{\mathrm{e}}$, and very low or zero variation. Such stars were not included in our catalog, partly because it was impossible to determine the magnetic period, $P_{\mathrm{mag}}$, and phases of individual $B_{\mathrm{e}}$ measurements. This paper is based on observational data published or posted in astronomical databases up to the end of August 2020.

Acknowledgements. We are grateful to John Landstreet and Stefano Bagnulo their comments and numerous suggestions. The authors thank D. Kurtz for providing the software program. Our research is based on data compiled and posted in the SIMBAD, ADS, and CDS databases.

\section{References}

Babcock, H. W. 1947, ApJ, 105, 105 Babcock, H. W. 1958, ApJS, 3, 141 Babcock, H. W. 1960, ApJ, 132, 521 Borra, E. F. 1981, ApJ, 249, L39

Borra, E. F., \& Landstreet, J. D. 1980, ApJS, 42, 421

Bychkov, V. D., Bychkova, L. V., \& Madej, J. 2005, A\&A, 430, 1143 Bychkov, V. D., Bychkova, L. V., \& Madej, J. 2016, MNRAS, 455, 2567 Hensberge, H., Maitzen, H. M., Deridder, G., et al. 1981, A\&AS, 46, 151 Mikulášek, Z. 2016, Contrib. Astron. Obs. Skaln. Pleso, 46, 95

Mikulášek, Z., \& Skarka, M. 2020, Contrib. Astron. Obs. Skaln. Pleso, 50, 430 Preston, G. 1971, ApJ, 164, 309

Romanyuk, I. I., Kudryavtsev, D. O., Semenko, E. A., \& Moiseeva, A. V. 2016, Astrophys. Bull., 71, 447

Sikora, J., David-Uraz, A., Chowdhury, S., et al. 2019, MNRAS, 487, 4695

Stibbs, D. W. N. 1950, MNRAS, 110, 395 